\title{
Comparaison par simulation de 3 types d'unités d'échantillonnage en futaies feuillues de hêtre (Fagus silvatica L.) (*)
}

\author{
J. HEBERT, J. RONDEUX, C. LAURENT * \\ Unité de Gestion et Economie forestières \\ * Centre de Recherche et de Promotion forestières \\ I.R.S.I.A. (Section "Aménagement et Production"), \\ Faculté des Sciences Agronomiques, 2, Passage des Déportés, Gembloux, 5800 Belgique
}

\begin{abstract}
Summary
Comparison between three types of sampling units in beech stands
\end{abstract}

\begin{abstract}
This study is dealing with the choice of the size and the type of sampling units which could be used in inventories of beech stands (Fagus silvatica L.). Two experimental areas of 16 hectares have been considered in evenaged and unevenaged structures.

Three types of sampling units were studied : fixed circular plots, Bitterlich plots (non fixed circular plots) and circular plots including a minimum of number of trees (15-20).

As regards to the estimations of basal area and number of trees per hectare there is no significative difference between plots of fixed area and plots including a minimum number of trees even if plot sizes and stand structure vary. As expected, compared to the other methods, the Bitterlich plots give little more precise estimations of basal area and less precise estimations of number of trees. The plots with a minimum number of trees would give higher values compared to the other types of plots but no significative difference have been observed.

Numerous simulations show that in case of a fixed total cost the use of a great number of small units or of a limited number of large units has little effect on the standard error of estimations.

The concept of minimum number of trees (15-20) seems to be valuable according to a compromise between precision and cost. To avoid a risk of bias in stands including large empty zones, it is preferable to use plot radii suitable to normal conditions.
\end{abstract}

Key words : Inventory, sampling units, beech stands.

\section{Résumé}

Trois types d'unités d'échantillonnage (placettes circulaires à surface définie, placettes Bitterlich à surface non définie et placettes à nombre minimum de tiges) ont été comparés au sein de dispositifs expérimentaux installés en hétraies.

$\left({ }^{*}\right)$ Etude financéc par l'Institut pour l'Encouragement de la Recherche Scientifique dans l'Industric et l'Agriculture (I.R.S.I.A.). 
De manic̀re générale, les calculs ont montré qu'il n’y avait pas de différences significatives entre les estimations des nombres de bois et des surfaces terrières par hectare, fournies par les types de placettes évoquées, quelles que soient leurs tailles et la structurc des peuplements. En ce qui concerne les précisions fournies par les trois méthodes, les estimations de la surface terrière donnent lieu à des précisions assez semblables avec un léger avantage toutefois à la méthode Bitterlich, alors que cette dernière s'avère moins précise que les deux autres dans le cas de restimation du nombre de bois par hectare.

L'étude, par simulation, de l'efficacité montre que pour un même effort d'échantillonnage ou un coût total fixé, le fait d'installer un nombre élevé de petites placettes ou un nombre limité de grandes placettes influence peu la précision obtenue quel que soit le type de placette considéré ou le paramètre estimé. Dans ces conditions, le choix de la taille de la placette se fera, avant tout, en fonction de contingences pratiques (visibilité, facilité d'installation...).

Enfin. si ce n’est pour la méthode Bitterlich. il existe une bonnc concordance entre les nombres de bois estimés par catégories de grosseur et ceux fournis par l'inventaire complet.

Les placettes à nombre minimum de tiges méritent d'être prises en considération pour autant qu'elles incorporent 15 à 20 hois. Si les peuplements inventoriés sont localement caractérisés par unc répartition très irrégulièrc des tiges et comportent de nombreuses trouées éparses ou des vides, il est recommandé, pour éviter des biais importants, de choisir des surfaces ou des rayons adaptés à des situations jugées normales ou moyennes au sein de ceux-ci.

Mots clés: Inventairé, échantillonnage, unités déchantillonnage, futaies de hêtre.

\section{Introduction}

Les inventaires forestiers par échantillonnage necessitent, outre le choix du plan d'inventaire, la définition précise de l'unité d'échantillonnage en ce qui concerne la grandeur de celle-ci et le mode de sélection des individus qui la constituent. Ces choix sont essentiellement liés à des contraintes de précision et de coût.

A partir de dispositifs expérimentaux installés dans le Sud-Est de la Belgique et déjà utilisés antérieurement pour des études de structure (LENGER, 1961) et d'échantillonnage de peuplements feuillus (Grayet, 1977), nous avons simulé 3 types d'échantillonnage au moyen de placettes circulaires à surface définie ou fixée, de placettes Bitterlich (à surface non définie) et de placettes circulaires à nombre minimum de tiges. Les comparaisons ont porté sur l'exactitude et la précision de l'estimation de diverses caractéristiques dendrométriques pour ces 3 modes de sélection d'arbres dans le cas de l'implantation d'unités de différentes tailles.

Le paragraphe 2 fournit une description du matériel expérimental, définit la nature et les principes de matérialisation des unités d'échantillonnage et présente les modalités testées ainsi que les variables étudiées. Le paragraphe 3 concerne les résultats obtenus, principalement ceux ayant trait à l'exactitude et à la précision des estimations réalisées. Enfin, le paragraphe 4 est consacré à quelques conclusions.

\section{Description du matériel expérimental et modalités d'échantillonnage testées}

\subsection{Matériel expérimental}

L'étude a porté sur l'analyse de données issues de deux dispositifs installés en futaies de hêtres et couvrant chacun 16 hectares, l'un en structure de type équienne, 
c'est-à-dire comportant très peu de surfaces régénérées, et l'autre en structure d'allure jardinée provenant de la transformation d'un taillis sous futaie.

Les coordonnées cartésiennes ainsi que les circonférences de tous les arbres situés dans ces dispositifs ont été enregistrées sur disque magnétique. Les données récoltées ont fait l'objet d'un traitement par ordinateur en vue de déterminer des nombres de bois, des surfaces terrières et des volumes ramenés à l'hectare et d'identifier la structure des peuplements par l'intermédiaire de la repartition des nombres de bois par catégories de circonférence à $1,5 \mathrm{~m}$ (fig. 1).

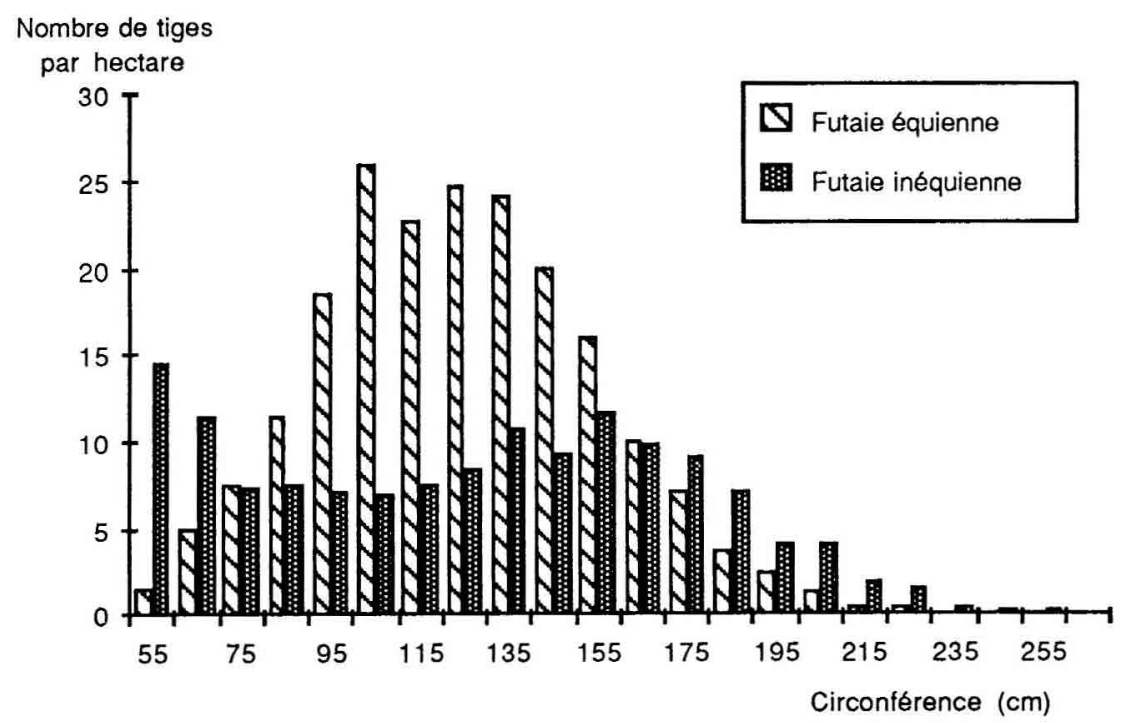

FIG. 1

Répartition des nombres de bois par hectare et par catégorie de grosseur pour chaque dispositif de 16 hectares.

Repartition of number of trees per hectare by girth classes.

\subsection{Définition et matérialisation des unités d'échantillonnage}

La définition des unités d'échantillonnage ou «placettes » testées se base sur des critères de surface, d'angle intercepté ou de nombre de bois, selon les cas.

Les placettes à surface définie ou fixée sont, pour des raisons pratiques, circulaires. Elles sont constituées de l'ensemble des arbres dont la distance au point de sondage est inférieure à une grandeur imposée. De ce fait, les arbres de différentes grosseurs sont sélectionnés avec une probabilité proportionnelle à leur fréquence d’observation.

Les placettes dites "Bitterlich" sont des unités à surface non définie. Tout arbre est inclus dans une unité s'il est vu sous un angle supérieur ou égal à un angle de balayage fixé, c'est-à-dire si son diamètre est au moins égal au produit de la tangente 
de cet angle par sa distance au point de sondage. La probabilité de sélection d'un arbre est proportionnelle à sa surface terrière.

Quant aux placettes à nombre minimum de tiges, elles sont délimitées à partir d'un rayon estimé à vue, de manière telle qu'au moins « $n$ » bois soient sélectionnés (Rondeux, 1985; Laurent et Rondeux, 1987). Il ne faut pas confondre ce type d'unités avec celles proposées par Prodan (1968), bien que dans ces deux cas, leur grandeur soit fonction des densités locales des peuplements (1).

\subsection{Mise en ceuvre des modalités d'échantillonnage testées et variables étudiées}

Pour comparer ces différentes méthodes, nous avons eu recours à un processus de simulation, ce qui impliquait la caractérisation aussi objective que possible des unités d'échantillonnage. Comme notre but était de tester les modes de sélection des arbres, nous avons adopté des conventions strictes quant à la mise en ouvre des 3 types de placettes, afin que le nombre de bois à mesurer par unité soit comparable. Nous avons considéré, lors de notre expérimentation, des valeurs entières du facteur de surface terrière (FST) "2) des placettes "Bitterlich » (LoETSCH et al., 1973; RondeUX, 1983). Pour chaque valeur de FST, le nombre moyen $\bar{n}$ de tiges par placette est donné par:

$$
\overrightarrow{\mathrm{n}}=\frac{\mathrm{G}}{\mathrm{FST}}
$$

où : $\mathrm{G}=$ surface terrière par hectare du dispositif.

$\bar{n}=$ nombre moyen de tiges par placette.

Les placettes à surface définie comportant en moyenne le même nombre de tiges auront donc pour surface (en ha) :

$$
\mathrm{s}=\frac{\overline{\mathrm{n}}}{\mathrm{N}}
$$

où $\mathrm{N}$ représente le nombre de tiges par hectare du dispositif.

La matérialisation des placettes à nombre minimum de tiges est beaucoup moins évidente car la technique utilisée sur le terrain suppose un «coup d'œil » qu'il faut tenter de simuler par le calcul. Lors d'une expérimentation réalisée préalablement sur le terrain et visant à mesurer la variabilité moyenne observée lors de l'estimation d'un rayon correspondant à une surface de placette devant en principe contenir au moins 15 bois, on a pu observer que le nombre de tiges par placette variait de 15 à 20 selon une distribution pouvant être assimilée à une loi exponentielle décroissante.

Comme le nombre moyen de tiges par placette était de 16 , nous avons fait l'hypothèse que la valeur du nombre minimum de tiges (nt) pouvait s'obtenir en multipliant par $15 / 16$ le nombre moyen de tiges $\bar{n}$ relatif à une placette à surface définie, soit :

$$
\mathrm{nt}=\overline{\mathrm{n}}(15 / 16)
$$

(1) La méthode du $6^{c}$ arbre de Prodan ne prend en compte que les 6 arbres les plus proches du point de sondage. Par convention, les 5 plus proches el la moitié du $6^{\circ}$ sont inclus dans une surface circulaire centrée sur le point de sondage, le rayon du cercle correspond à la distance séparant le 6 arbre de ce même point.

(2) FST = facteur de surface terrière ou nombre par lequel il faut multiplier le nombre de tiges interceptées en un tour d'horizon complet pour obtenir la surface terrière estimée à l'hectare. 
Afin de simuler le nombre de tiges (nr) réellement incluses dans la placette, l'appréciation visuelle de l'opérateur est obtenue en ajoutant au nombre minimum fixé (nt) un nombre aléatoire qui est fonction du nombre de tiges à sélectionner et de la distribution exponentielle des nombres de tiges par placettes observée lors de l'expérimentation préalable sur le terrain (avec nt $=15$ ). Nous sommes arrivés ainsi au résultat empirique suivant :

$$
\mathrm{nr}=\mathrm{nt}+\text { partie entière de }\left(\frac{\mathrm{y} \cdot \mathrm{nt}}{15}\right)
$$

où y est une variable aléatoire exponentielle de moyenne égale à 1 et dont le domaine de variation est compris entre 0 et 6 .

Pour éviter un biais, le rayon de la placette correspond à une valeur choisie au hasard entre la distance séparant le centre de la placette du $n^{\text {iènle }}$ bois et la distance de ce même centre au $(\mathrm{nr}+1)^{\text {ieme }}$ bois.

Afin de comparer les diverses modalités d'échantillonnage, et compte tenu de la surface des dispositifs, nous avons considéré 49 unités d'échantillonnage réparties de manière systématique. Ce nombre permet d'éviter un problème de chevauchement d'unités eu égard à la taille maximale que nous souhaitions leur conférer et à la dimension, très variable d'un endroit à l'autre, des «placettes » Bitterlich.

Pour chacun des 3 types d'unités étudiées, nous avons imposé 4 tailles correspondant respectivement aux facteurs de surface terrière $1,2,3$ et 4 et induisant des valeurs moyennes de surface de placettes ou des moyennes de nombres de bois liées à la structure des peuplements et à la distribution spatiale des arbres. Le tableau 1 indique. pour les 2 dispositifs, les grandeurs moyennes des placettes induites par les facteurs de surface terrière préconisés.

TABLEAU 1

Correspondance entre les tailles des 3 types d'unités d'échantillonnage. Correspondance between the sizes of the 3 sampling units.

\begin{tabular}{|c|c|c|c|c|}
\hline Futaie équienne & & & & \\
\hline Bitterlich (FST) $\ldots \ldots \ldots$ & $1 \mathrm{~m}^{2} / \mathrm{ha}$ & $2 \mathrm{~m}^{2} / \mathrm{ha}$ & $3 \mathrm{~m}^{2} / \mathrm{ha}$ & $4 \mathrm{~m}^{2} / \mathrm{ha}$ \\
\hline Surface définie (ares) & 12,81 & 6,41 & 4,27 & 3,20 \\
\hline Nombre minimum de tiges & 24 & 12 & 8 & 6 \\
\hline Futaie d'allure jardinée & & & & \\
\hline Bitterlich (FST) & $1 \mathrm{~m}^{2} / \mathrm{ha}$ & $2 \mathrm{~m}^{2} / \mathrm{ha}$ & $3 \mathrm{~m}^{2} / \mathrm{ha}$ & $4 \mathrm{~m}^{2} / \mathrm{ha}$ \\
\hline Surface définie (ares) & 14,56 & 7,28 & 4,85 & 3,64 \\
\hline Nombre minimum de tiges & 19 & 10 & 6 & 5 \\
\hline
\end{tabular}

Dans ces conditions, les comparaisons porteront sur 12 cas (3 types d'unités $\times 4$ tailles) aussi bien pour les nombres de bois et les surfaces terrières par hectare que pour les nombres de bois par catégories de circonférence à $1,5 \mathrm{~m}$ du sol. 


\section{Résultats obtenus}

Nous avons successivement comparé, pour les trois types d'unités, l'exactitude et la précision de l'estimation des paramètres globaux ramenés à l'hectare. En outre, nous nous sommes également préoccupés de déterminer l'efficacité des méthodes testées. Dans le cas de l'estimation de la répartition des nombres de bois par catégories de grosseur, nous avons uniquement tenté de faire ressortir les principales tendances.

1) En ce qui concerne l'exactitude, nous avons cherché à savoir d'une part si les estimations moyennes d'un même paramètre fournies par les 3 méthodes pouvaient être considérées comme égales et, d’autre part, si ces mêmes estimations moyennes ne s'écartaient pas trop des valeurs de référence fournies par l'inventaire complet du matériel.

Dans chaque dispositif, nous avons procédé, pour chacune des variables étudiées, à la comparaison de 4 groupes de 3 moyennes, chaque groupe étant associé à une dimension de placette déterminée à partir des facteurs de surface terrière. Les résultats relatifs aux moyennes et aux variances estimées des nombres de bois et des surfaces terrières à l'hectare sont fournis dans les tableaux 2 et 3.

\section{TABIHAU 2}

Moyennes des estimations selon les dispositifs et les types d'unités testées

$(B-$ Biturlich, $S D=$ sarface définie, $N M=$ nombre minimum de liges. $I C=$ inventaire complet).

Meams of estimations (for number of trees and basal areas) related to experimental areas and rypes of units $(B=$ Bitterlich,$S D=$ defined of fixed area, $N M=$ minimum number of trees. $I C=$ complete enumeration).

Nombre de bois a lhoctare

\begin{tabular}{|c|c|c|c|c|c|c|}
\hline & \multicolumn{3}{|c|}{ Futaic équienne $(I C=20-4,0)$} & \multicolumn{3}{|c|}{ Futaie d’allure jardinée $(\mathrm{IC}=142,4)$} \\
\hline & B & SD & NM & B & $\mathrm{SD}$ & NM \\
\hline FST 1 & 206,7 & 209.8 & 209,5 & 146.4 & 139,4 & 141,9 \\
\hline FST 2 & 211.9 & 205.1 & 211.1 & 154.9 & 142.4 & 149.9 \\
\hline FST 3 & 203,9 & 210,7 & 215,0 & 155,2 & 145,6 & 157,4 \\
\hline FST 4 & 208.9 & 204.2 & 214.8 & 161,9 & 142.4 & 161.2 \\
\hline
\end{tabular}

Surface terriere à lihectare

\begin{tabular}{|c|c|c|c|c|c|c|}
\hline & \multicolumn{3}{|c|}{ Futaic Équicnne $\left(\mathrm{IC}=26.13 \mathrm{~m}^{2}\right)$} & \multicolumn{3}{|c|}{ Futaic d'allure jardinéc (IC $=20,74 \mathrm{~m}^{2}$} \\
\hline & B & SD & NM & B & $\mathrm{SD}$ & NM \\
\hline FST 1 & 27.24 & 27.39 & 27.35 & 21,22 & 21.26 & 21,14 \\
\hline FST 2 & 27,55 & 26.57 & 27.11 & 21,63 & 21,66 & 21,97 \\
\hline FSI 3 & 26.20 & $27.60)$ & 28.24 & 21,86 & 21.47 & 21.62 \\
\hline FST 4 & 27.35 & 26.54 & 27,93 & 22.12 & 20,69 & 21,97 \\
\hline
\end{tabular}




\section{TABLEAU 3}

Comparaisons deux à deux des variances relatives aux estimations des nombres de bois selon les différents types d'unités testées $(B=$ Bitterlich, $S D=$ surface définie,

$N M=$ nombre minimum de tiges).

Comparison of variances related to estimations of number of trees according to the various kinds of sampling units.

\begin{tabular}{c|c|c}
\hline & Futaie équienne & Futaie d'allure jardinée \\
\cline { 2 - 3 } FST 1 & $\sigma_{\mathrm{SD}}^{2}=\sigma_{\mathrm{NM}}^{2}=\sigma_{\mathrm{B}}^{2}$ & $\sigma_{\mathrm{SD}}^{2}=\sigma_{\mathrm{NM}}^{2}<\sigma_{\mathrm{B}}^{2}$ \\
FST 2 & $\sigma_{\mathrm{SD}}^{2}=\sigma_{\mathrm{NM}}^{2}=\sigma_{\mathrm{B}}^{2}$ & $\sigma_{\mathrm{SD}}^{2}<\sigma_{\mathrm{NM}}^{2}<\sigma_{\mathrm{B}}^{2}$ \\
& $\left(\right.$ mais $\left.\sigma_{\mathrm{S}}^{2}<\sigma_{\mathrm{B}}^{2}\right)$ & \\
FST 3 & $\sigma_{\mathrm{SD}}^{2}=\sigma_{\mathrm{NM}}^{2}<\sigma_{\mathrm{B}}^{2}$ & $\sigma_{\mathrm{SD}}^{2}<\sigma_{\mathrm{NM}}^{2}<\sigma_{\mathrm{B}}^{2}$ \\
FST 4 & $\sigma_{\mathrm{SD}}^{2}=\sigma_{\mathrm{NM}}^{2}=\sigma_{\mathrm{B}}^{2}$ & $\sigma_{\mathrm{SD}}^{2}<\sigma_{\mathrm{NM}}^{2}<\sigma_{\mathrm{B}}^{2}$ \\
\hline
\end{tabular}

Les tests d'égalité des moyennes réalisés par l'analyse de la variance ne permettent pas d'affirmer qu’il existe des différences significatives entre les estimations liées au mode de sélection des arbres. Toutefois, on peut noter que les placettes à nombre minimum de tiges ont tendance à engendrer des surestimations du nombre de bois lorsque la taille des placettes diminue. Il parait raisonnable de choisir un nombre de tiges supérieur à 10 pour limiter l'occurrence d’un biais.

2) Afin de comparer les précisions des 3 méthodes, nous avons confronté les variances des estimations qu'elles fournissent. Pour tenir compte de la corrélation entre les estimations, nous avons appliqué le test de conformité du coefficient de la droite des moindres rectangles (hypothèse nulle $\gamma=1$ ), en considérant les 3 méthodes prises deux à deux, et ce pour chaque dimension de placette.

Le tableau 3 montre que pour l'estimation du nombre de bois à lhectare, la méthode Bitterlich se distingue presque toujours par une précision moindre surtout en futaie inéquienne. Par contre, dans le cas de l'estimation de la surface terrière (tabl. 4), les 3 méthodes ont dans l'ensemble une précision assez semblable avec toutefois un léger avantage pour la méthode Bitterlich.

\section{TABLEAU 4}

Comparaisons deux à deux des variances relatives aux estimations des surfaces terrières selon les différents types d'unités testées $(B=$ Bitterlich, $S D=$ surface définie.

$N M=$ nombre minimum de tiges).

Comparison of variances related to estimations of basal area according to the various kinds of sampling units.

\begin{tabular}{c|c|c}
\hline & Futaic équienne & Futaie d'allure jardinée \\
\cline { 2 - 3 } FST 1 & $\sigma_{\mathrm{SD}}^{2}=\sigma_{\mathrm{NM}}^{2}=\sigma_{\mathrm{B}}^{2}$ & $\sigma_{\mathrm{SD}}^{2}=\sigma_{\mathrm{NM}}^{2}=\sigma_{\mathrm{B}}^{2}$ \\
FST 2 & $\sigma_{\mathrm{S}}^{2}=\sigma_{\mathrm{NM}}^{2}=\sigma_{\mathrm{B}}^{2}$ & $\sigma_{\mathrm{SD}}^{2}=\sigma_{\mathrm{NM}}^{2}=\sigma_{\mathrm{B}}^{2}$ \\
FST 3 & $\sigma_{\mathrm{S}}^{2}=\sigma_{\mathrm{NM}}^{2}=\sigma_{\mathrm{B}}^{2}$ & $\sigma_{\mathrm{SD}}^{2}=\sigma_{\mathrm{NM}}^{2}=\sigma_{\mathrm{B}}^{2}$ \\
FST 4 & $\sigma_{\mathrm{SD}}^{2}=\sigma_{\mathrm{NM}}^{2}>\sigma_{\mathrm{B}}^{2}$ & $\sigma_{\mathrm{SD}}^{2}=\sigma_{\mathrm{NM}}^{2}>\sigma_{\overline{\mathrm{B}}}^{2}$ \\
\hline
\end{tabular}


3) Nous avons en outre voulu déterminer, parmi les 12 unités testées, celle qui offre l'efficacité relative la plus élevée c'est-à-dire celle qui, pour un coût total fixé, fournit la précision la plus élevée ou encore l'erreur-standard de la caractéristique moyenne (nombre de bois $\mathrm{N}$ ou surface terrière $\mathrm{G}$ ) la plus faible.

L'erreur-standard de la moyenne (ou son carré) est estimée comme suit :

$$
\hat{\sigma}_{\overline{\mathrm{x}}}^{2}=\frac{\hat{\sigma}^{2}}{\mathrm{p}}(1-\mathrm{f}),
$$

où $\hat{\sigma}^{2}=$ estimation de la variance des estimations des caractéristiques ( $N$ ou $\left.G\right)$ liée au type et à la taille des placettes,

$f=$ taux de sondage ou fraction sondée,

$\mathrm{p}=$ nombre d'estimations ou nombre de placettes.

Si en outre, NS désigne le nombre total de bois sélectionnés et $\bar{n}$ le nombre moyen de bois par placette, on peut décrire que :

$$
\mathrm{NS}=\mathrm{p} \cdot \overline{\mathbf{n}} \text {. }
$$

Si NT désigne le nombre total de bois du peuplement et si on admet qu'il existe, pour un type de placette choisi et une caractéristique estimée ( $N$ ou $G$ ), une relation du type :

$$
\hat{\sigma}^{2}=\frac{\mathrm{a}}{(\overline{\mathrm{n}})^{\mathrm{h}}}
$$

a et b étant 2 paramètres estimés par régression, on peut aussi écrire que :

$$
\hat{\sigma}_{\overline{\mathrm{x}}}^{2}=\frac{a \bar{n}}{N S(\overline{\mathrm{n}})^{\mathrm{b}}}\left(1-\frac{\mathrm{NS}}{\mathrm{NT}}\right),
$$

ou encore que :

$$
\hat{\boldsymbol{\sigma}}_{\overline{\mathrm{x}}}^{2}=\frac{\mathrm{a}}{(\overline{\mathrm{n}})^{\mathrm{b}-1}}\left(\frac{1}{\mathrm{NS}}-\frac{1}{\mathrm{NT}}\right)
$$

qui s’écrira :

$$
\hat{\sigma}_{\dot{\mathrm{x}}}^{2}=\frac{\mathrm{a}}{\mathrm{NS}(\overline{\mathrm{n}})^{\mathrm{b}} \quad 1}
$$

la fraction $\frac{1}{\mathrm{NT}}$ étant tout à fait négligeable dans le cas d'inventaires relatifs à de grands peuplements.

Les tableaux 5 et 6 fournissent les résultats relatifs aux variances estimées des nombres de bois et des surfaces terrières ainsi que les valeurs estimées des paramètres a et $b$.

D'autre part, le coût total, qui peut par exemple s'exprimer en temps nécessaire pour réaliser l'échantillonnage, se décompose en deux parties:

- le temps requis pour effectuer les mesures proprement dites qui est proportionnel au nombre d'arbres mesurés,

- le temps requis pour cheminer dans le peuplement forestier en reliant tous les points de sondage. 


\section{TABleau 5}

Variances estimées des nombres de bois/ha selon 4 facteurs de surface terrière et valeurs estimées des paramètres a et $b$.

Estimated variances of number of trees per hectare according to 4 basal area factors and estimated values of $a$ and $b$ parameters.

\begin{tabular}{c|c|c|c|c|c|c}
\hline & \multicolumn{3}{|c|}{ Futaie équienne } & \multicolumn{3}{c}{ Futaie d'allure jardinée } \\
\cline { 2 - 7 } & $\begin{array}{c}\text { Méthode } \\
\text { Bitterlich }\end{array}$ & $\begin{array}{c}\text { Surface } \\
\text { définie }\end{array}$ & $\begin{array}{c}\text { Nb min. } \\
\text { de tiges }\end{array}$ & $\begin{array}{c}\text { Méthode } \\
\text { Bitterlich }\end{array}$ & $\begin{array}{c}\text { Surface } \\
\text { définie }\end{array}$ & $\begin{array}{c}\text { Nb min. } \\
\text { de tiges }\end{array}$ \\
\cline { 2 - 7 } FST 1 & 1654 & 1598 & 1558 & 3857 & 1083 & 1113 \\
FST 2 & 3115 & 1987 & 2432 & 7727 & 2013 & 3043 \\
FST 3 & 4719 & 2524 & 2137 & 10354 & 3418 & 5514 \\
FST 4 & 4198 & 4402 & 5210 & 14490 & 5033 & 8085 \\
$\hat{\mathrm{a}}$ & 20000 & 12000 & 20000 & 66000 & 29000 & 87000 \\
$\hat{\mathrm{b}}$ & 0.75 & 0,66 & 0.79 & 0,93 & 1,10 & 1,44 \\
\hline
\end{tabular}

Tableau 6

Variances estimées des surfaces terrières/ha selon 4 factears de surface terrière. Estimated variances of basal areas per hectare according to 4 basal area factors and estimated values of $a$ and $b$ parameters.

\begin{tabular}{c|c|c|c|c|c|c}
\hline & \multicolumn{3}{|c|}{ Futaic équienne } & \multicolumn{3}{c}{ Futaic d’allure jardinćc } \\
\cline { 2 - 7 } & $\begin{array}{c}\text { Méthode } \\
\text { Bitterlich }\end{array}$ & $\begin{array}{c}\text { Surface } \\
\text { définie }\end{array}$ & $\begin{array}{l}\text { Nb min. } \\
\text { de tiges }\end{array}$ & $\begin{array}{c}\text { Méthode } \\
\text { Bitterlich }\end{array}$ & $\begin{array}{c}\text { Surface } \\
\text { définie }\end{array}$ & $\begin{array}{c}\text { Nb min. } \\
\text { de tiges }\end{array}$ \\
\cline { 2 - 7 } FST 1 & 11,61 & 12,71 & 11,13 & 16,89 & 14,13 & 11,82 \\
FST 2 & 18,54 & 20,97 & 21,53 & 23,28 & 20,90 & 27,03 \\
FST 3 & 32,54 & 32,38 & 34,37 & 39,38 & 43,66 & 33,45 \\
FST 4 & 29,56 & 49.62 & 70,13 & 41,40 & 72,39 & 77,18 \\
$\hat{a}$ & 140 & 270 & 620 & 130 & 430 & 480 \\
$\hat{b}$ & 0,76 & 0,96 & 1,26 & 0,70 & 1,17 & 1,24 \\
\hline
\end{tabular}

La première composante peut s'écrire :

$$
\mathrm{T}_{\mathrm{t}}=\mathbf{N S} \mathbf{t}_{\mathrm{i}} \text {, }
$$

où $\mathrm{t}_{\mathrm{i}}$ désigne le temps moyen consacré aux mesures réalisées sur un arbre.

La deuxième composante est beaucoup plus difficile à maitriser. Elle dépend très largement de la taille, de la localisation topographique et de la forme des peuplements échantillonnés ; elle dépend également du nombre de placettes et du plan d'échantillonnage choisi. Pour simplifier, on supposera un échantillonnage systématique à mailles carrées. En première approximation, la deuxième composante peut s'écrire : 


$$
T_{2}=\sqrt{p} t_{i l}
$$

où $t_{\sqrt{ }}$ désigne le temps nécessaire pour parcourir, par convention. la diagonale du peuplement forestier avec les moyens choisis pour cheminer d'une placette à l'autre (boussole, mesureur de distance).

Par conséquent. le temps total $\mathrm{T}$ peut sécrire :

$$
\mathrm{T}=\mathrm{NS} \mathrm{t}_{\mathrm{i}}+\sqrt{\mathrm{p}} \mathrm{t}_{\mathrm{i}}
$$

ou encore :

$$
T=N S t_{i}+\sqrt{\frac{N S}{\bar{n}}} t_{i} \cdot
$$

De la relation précédente (équation du second degré en $\sqrt{\mathrm{NS}}$ ), on peut déduire la valeur de NS compatible avec un coût total $T$ fixé. On trouve ainsi :

$$
N S=\frac{2 T^{2}}{\frac{t_{11}^{2}}{\bar{n}}+2 t_{1} T+\sqrt{\frac{t_{d 1}^{2}}{\bar{n}}\left(\frac{t_{\bar{u}}^{2}}{\bar{n}}+4 t_{i} T\right)}}
$$

En pratique, on se fixe comme contraintes des valeurs de $t_{i}, t_{j}, T$ et on choisit plusieurs valeurs de $\bar{n}$ (nombre moyen de bois par placette), ce qui conduit à plusieurs évaluations du nombre total de bois sélectionnés NS. Ensuite, pour chacune des valeurs de NS compatibles avec un coût total préfixé T, on estime les différentes valeurs de $\hat{\sigma}_{\bar{x}}^{2}$ selon le type de placette et selon la caractéristique dendrométrique envisagée ( $N$ ou $G$ ).

On peut ainsi trouver le type et la taille de la placette qui, pour un coût fixé, conduit à l'estimation la plus précise.

Différentes simulations réalisées sur cette base ont montré que la variance estimée passe effectivement par un minimum mais que ce minimum est très peu marqué dans une large mesure quel que soit le nombre total d'arbres à mesurer et le type de placette. Par conséquent, il convient plutôt de choisir une taille de placette en fonction de contingences pratiques (visibilité, accès, etc.).

4) Dans le prolongement des calculs effectués, nous avons également tenté de voir si l'on peut, dans les conditions de l'étude, s'attendre à des résultats comparables en ce qui concernait la ventilation des nombres de bois à l'hectare par catégories de gestion ( $<60 \mathrm{~cm}$ de circonférence, 60 à $89 \mathrm{~cm}, 90$ à $119 \mathrm{~cm}, 120$ à $149 \mathrm{~cm}, 150$ à $179 \mathrm{~cm}$, $>179 \mathrm{~cm}$ ). Les résultats obtenus font l'objet du tableau 7 .

Dans l'ensemble, on peut constater qu'il existe une assez bonne concordance entre l'allure des distributions de fréquence théoriques (inventaire complet) et celle des distributions de fréquence observées relatives aux diverses modalités de sélection et tailles d'unités. Les meilleurs résultats sont néanmoins observés pour les unités de tailles élevées et plus spécialement celles à surfaces définies. Comme il fallait s'y attendre, la méthode Bitterlich donne lieu aux plus grandes différences avec l'inventaire complet et montre une assez grande variabilité d'un facteur de surface terrière à l'autre. Quant aux placettes à nombre minimum de tiges qui paraissaient dignes d'intérêt dans l'estimation des paramètres globaux (nombres de bois et surfaces terrières à l'hectare), elles donnent des résultats satisfaisants pour autant que le nombre de bois pris en considération soit au moins égal à 10 , ce qui implique, dans nos conditions de travail, le choix d'un rayon atteignant, en moyenne, une quinzaine de mètres ( $\sim 7$ ares). 


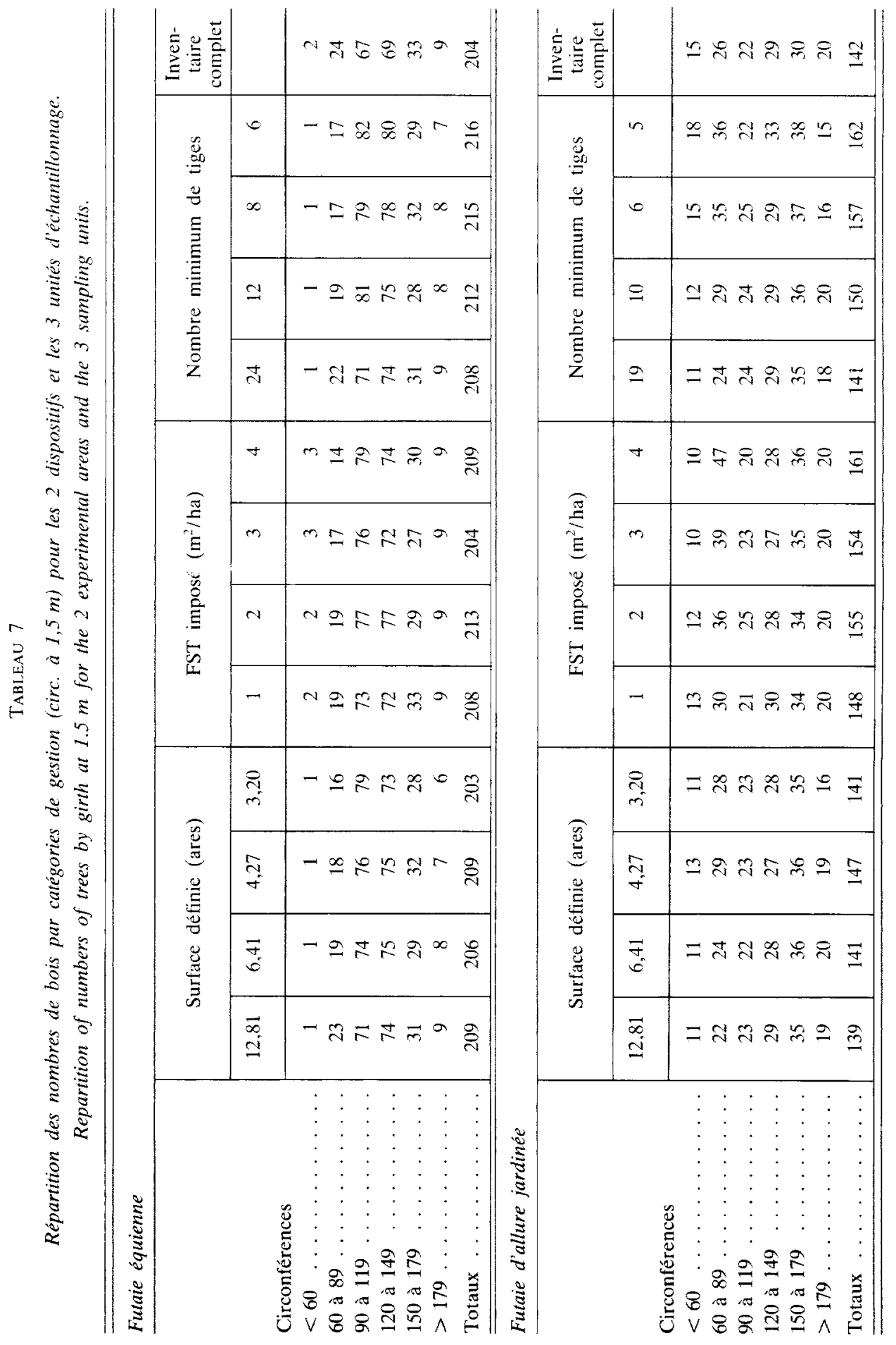




\section{Conclusions}

L'étude entreprise sur la base de 2 dispositifs expérimentaux installés en futaie feuillue de hêtre visait à comparer 3 types d'unités d'échantillonnage définies par le mode de sélection des arbres. Nous avons comparé des placettes à surface définie ou fixée, des placettes Bitterlich (à surface non définie) et des placettes à nombre minimum de tiges, ces dernières étant définies par un rayon tel que le nombre de bois compris dans l'aire circulaire correspondantc atteigne au moins une valeur prédéterminée.

En ce qui concerne le nombre de bois et la surface terrière à l'hectare, les calculs ont montré qu'il n'y a pas de différence significative entre les estimations réalisées à partir des différents types de placette, quelles que soient leurs tailles et quelle que soit la structure du peuplement.

Les placettes à nombre minimum de tiges auraient tendance, et ce d'autant plus que ce nombre est réduit, à fournir des estimations du nombre de bois plus élevées que les 2 autres types de placettes sans être pour autant significativement différentes.

En effectuant les comparaisons 2 à 2 des variances obtenues, nous avons constaté qu'elles peuvent être considérées comme identiques dans l'estimation de la surface terrière, si ce n'est pour les plus petites placettes et qu'elles étaient beaucoup moins stables dans l'estimation du nombre de bois, plus spécialement dans le peuplement d'allure jardinée, la méthode de Bitterlich se distinguant par sa précision inférieure.

En ce qui concerne l'efficacité des différentes méthodes envisagées, vue sous l'angle de leur transposition en conditions réelles d'inventaire, il est évident que les temps et donc les coûts liés aux opérations de mesure et aux déplacements sont déterminants. De nombreuses simulations portant sur les nombres de bois à mesurer par placette, les temps de déplacement et de mesure montrent, dans les conditions de notre expérimentation, que l'erreur-standard minimale n'est pas en relation étroite avec le nombre d'arbres mesurés et le type de placette pour un même effort d'échantillonnage.

Enfin, si on s'intéresse à la qualité des estimations des nombres de bois par catégories de grosseur, fournies par les diverses méthodes, un examen rapide des moyennes obtenues révèle une concordance satisfaisante avec les résultats issus des inventaires complets. Comme il fallait s'y attendre, les résultats les plus satisfaisants sont obtenus avec les placettes à surface définie de dimensions élevées. Des résultats satisfaisants peuvent encore être obtenus par l'intermédiaire de placettes dont le minimum de bois serait supérieur à 10 . Quant aux placettes Bitterlich, elles donnent lieu à des différences assez sensibles.

On peut déduire de ces quelques considérations que les placettes comportant un minimum de 15 à 20 bois constituent souvent un bon compromis entre la précision et la rapidité d'installation, ce qui plaiderait, mais cela doit encore être vérifié, en faveur de placettes à nombre de tiges relativement constant et donc à surface adaptée à la densité des peuplements. On notera en effet que cette méthode devra être utilisée avec beaucoup de précaution dans le cas de peuplements qui présenteraient de nombreuses trouées éparses et dans toutes les situations où la mesure d'un nombre minimum de bois supposerait que l'on adopte un rayon de placette anormalement élevé. Pour éviter ou diminuer les risques de biais, on choisira comme rayon celui qui aurait été établi au sein des zones à densités moyennes « normales » de ces peuplements. 
Il convient d'attirer l'attention sur le fait que les résultats obtenus concernent exclusivement des dispositifs expérimentaux et que leur extrapolation à des situations très particulières implique certaines réserves. Néanmoins, étant donné la structure des peuplements analysés, par rapport à celles des hêtraies couvrant le Sud de la Belgique, il nous paraît que les grandes tendances traduites par les résultats devraient être confirmées dans l'hypothèse où les méthodes préconisées seraient appliquées à l'inventaire d'autres peuplements.

Reçu le 23 septembre 1987.

Accepté le 15 février 1988.

\section{Références bibliographiques}

Grayer J.P., 1977. Contribution à l'étude de l'échantillonnage en futaie feuillue. Ann. Sci. Forest., 34 (1), 59-75.

Laurent C. et Rondeux J., 1987. Etude comparative de diverses unités d'échantillonnage en futaies feuillues de hêtres (Fagus silvatica L.). Document 86-1. Centre de Recherche et de Promotion Forestières. I.R.S.I.A.. Section «Aménagement et Production», Fac. Sci. Agron.. Gembloux, $16 \mathrm{p}$.

Lenger A., 1961. Etude de la répartition des arbres dans une futaie feuillue. Paris, $33^{c}$ session. Bull. Inst. Int. Stat., 5 p.

Loetsch F., Zöhrer F., HAller H., 1973. Forest inventory. Vol. 2. BLV. Verlagsgesellschaft. München, $469 \mathrm{p}$.

Prodan M., 1968. Punktstichprobe für die Forsteinrichtung. Forst. u. J. Holzw. 23 (11), 225-226.

Rondeux J., 1983. La méthode de l'angle critique : une conception particulière de l'échantillonnage appliqué aux inventaires forestiers. Annales de Gembloux, 89 (3), 183-200.

Rondeux J., 1985. An outline of a regional forest inventory in Belgium. Mitt. d. Abt. f. Forstl. Biometrie. Univ. Freiburg i. Br., 85 (3), 163-169. 